\title{
Interaction of Nitrogen Plasmas with Tungsten
}

\author{
K. Schmid*, A. Manhard, Ch. Linsmeier, A. Wiltner, T. \\ Schwarz-Selinger, W. Jacob, S. Mändl ${ }^{\dagger}$
}

Max-Planck-Institut für Plasmaphysik, EURATOM Association, Boltzmannstraße 2, 85748 Garching b. München, Germany

† Leibniz-Institut für Oberflächenmodifizierung, Permoserstr. 15, 04318 Leipzig, Germany

PACS numbers: 61.80.Jh, 52.77.Dq, 52.70.Nc, 52.40.Hf

\begin{abstract}
.
The use of nitrogen seeding to reduce the edge plasma temperature has recently been successfully applied in ASDEX Upgrade. While the plasma performance was significantly improved compared to other seeding species like $\mathrm{Ar}$ or $\mathrm{Ne}$, questions remained as to the interaction of nitrogen with a tungsten first wall. In particular the formation of thick tungsten nitride layers with reduced melting temperature and increased physical sputtering was a concern. Therefore dedicated laboratory experiments have been performed to investigate the interaction of $\mathrm{W}$ surfaces with $\mathrm{N}$ plasmas. Tungsten coated Si samples were exposed to $\mathrm{N}$ ions from plasma and ion gun sources at energies from $20 \mathrm{eV}$ to $10 \mathrm{keV}$ and $\mathrm{W}$ surface temperatures from 300 to $750 \mathrm{~K}$. After exposure to the $\mathrm{N}$ plasma with fluences of up to several $10^{23} \frac{\mathrm{N}}{\mathrm{m}^{2}}$ the $\mathrm{N}$ content in the samples was measured by nuclear reaction analysis. The sputter erosion was determined by measuring the thickness change of the $\mathrm{W}$ layer by RBS. The formation of $\mathrm{W}$ nitride phases was investigated in separate XPS experiments where the samples were implanted in situ with $\mathrm{keV} \mathrm{N}$ ions. It was found that only very small fractions of $\mathrm{N}$ are accumulated in the $\mathrm{W}$ surface and that $\mathrm{N}$ is bound in a nitride state. At temperatures above $600 \mathrm{~K}$ the nitrides are no longer stable which further reduces the $\mathrm{N}$ uptake into the $\mathrm{W}$. Moreover the accumulation of $\mathrm{N}$ in the surface leads to a decrease in $\mathrm{W}$ physical sputtering due to the lower $\mathrm{W}$ concentration at the surface.
\end{abstract}

* Corresponding author (Klaus.Schmid@ipp.mpg.de) 


\section{Introduction}

With the transition to a full $\mathrm{W}$ coated first wall in ASDEX Upgrade the lack of radiation from $\mathrm{C}$ in the edge plasma has led to an increase of the power flux to the outer target making it necessary to seed impurities into the edge plasma for radiative cooling [1]. Besides several noble gases also $\mathrm{N}$ was used since it is a more pronounced edge and divertor plasma radiator compared to $\mathrm{Ne}$ or $\mathrm{Ar}$ in AUG. While it turned out that $\mathrm{N}$ seeding not only efficiently cooled the edge plasma but also improved the overall plasma performance [1], questions remained about effects of $\mathrm{N}$ on the $\mathrm{W}$ plasma-facing components. In particular the formation of nitrides with different thermodynamic properties (lower melting temperature, higher sublimation) and the possibility of enhanced physical sputtering was a concern. Furthermore it was unknown whether large amounts of $\mathrm{N}$ would accumulate in the first wall which could then be released in subsequent discharges, potentially leading to problems in density control.

Since very little is known about the interaction of $\mathrm{N}$ plasmas with $\mathrm{W}$ surfaces and about the thermodynamics of the $\mathrm{N}-\mathrm{W}$ system, dedicated experiments were performed. These experiments were focussed in particular on the following processes: $\mathrm{N}$ accumulation in $\mathrm{W}$ as function of implantation energy, fluence and temperature, sputtering of $\mathrm{W}$ by $\mathrm{N}$ as function of energy and formation of nitride phases during implantations.

These experiments were performed at different experimental setups: The $\mathrm{N}$ accumulation as function of energy and fluence was investigated in a low pressure steady state electron cyclotron resonance (ECR) plasma chamber. Additionally, the temperature dependence of the $\mathrm{N}$ accumulation in $\mathrm{W}$ was investigated in a plasma immersion implantation setup at the IOM in Leipzig. The formation of nitride phases was investigated in an XPS setup where $\mathrm{W}$ was implanted with $\mathrm{keV} \mathrm{N}$ ions in a UHV environment. In addition to the experimental work, a phase diagram of the $\mathrm{N}-\mathrm{W}$ system was calculated based on the little available thermodynamic data.

\section{Thermodynamic data of the nitrogen tungsten system}

In equilibrium the amount of $\mathrm{N}$ retained in $\mathrm{W}$ due to ion implantation depends on the implantation depth, which is determined by the $\mathrm{N}$ energy, the solubility of $\mathrm{N}$ in $\mathrm{W}$ and the rate at which $\mathrm{N}$ diffuses into the $\mathrm{W}$ bulk and out of the sample. Low diffusivity limits $\mathrm{N}$ accumulation to the implantation zone which is saturated with N. For the particle energies involved $(<100 \mathrm{eV})$ the implantation zone is $<10 \mathrm{~nm}$. A high diffusivity on the other hand would lead to accumulation of large amounts of $\mathrm{N}$, since the implanted $\mathrm{N}$ can diffuse into the bulk, away from the quickly saturated implantation zone. In [2] the diffusion of $\mathrm{N}$ in $\mathrm{W}$ is given by Eq. 1

$$
\begin{aligned}
& D(T)=4.3 \times 10^{-4} \exp ^{\frac{-2.3}{K_{B}}}\left(\frac{m^{2}}{s}\right) \\
& K_{B}=\text { Boltzmann Constant }(\mathrm{eV} / \mathrm{K}) \\
& T \quad=\text { Temperature }(\mathrm{K})
\end{aligned}
$$


The diffusivity in Eq. 1 is negligible for temperatures well below $600 \mathrm{~K}$. The solubility of $\mathrm{N}$ in $\mathrm{W}$ depends on the thermodynamic phase in which the implanted $\mathrm{N}$ is retained. The phase with the highest $\mathrm{N}$ content is the WN phase which contains $50 \% \mathrm{~N}$.

The published data on thermodynamic properties of the $\mathrm{N}-\mathrm{W}$ system is sparse: Several crystallographic phases were identified and the solubility of $\mathrm{N}$ in $\mathrm{W}$ was measured in the temperature range from $2473 \mathrm{~K}$ to $3073 \mathrm{~K}[3,4,5,6]$. All the available data are contained in the SSOL4 data base which is part of the ThermoCalc ${ }^{\mathrm{TM}}[7]$ modeling package. The phase diagram calculated by ThermoCalc based on the available data is shown in Fig. 1. During the phase diagram calculation the gaseous $\mathrm{N}_{2}$ phase was excluded such that only the solid and liquid phases appear in the phase diagram. This corresponds to the experimental condition where the ambient $\mathrm{N}_{2}$ pressure is kept high enough such that it is always the phase with highest Gibbs enthalpy, i.e. it is not the thermodynamically stable phase. The phase diagram shows two nitride phases, namely the $\mathrm{WN}$ and $\mathrm{W}_{2} \mathrm{~N}$ phases. For the temperature range accessible in our experiment one would therefore expect that predominantly the WN phase is formed.

When the $\mathrm{N}_{2}$ gas phase is included in the calculations at pressures of $10^{5} \mathrm{~Pa}$ and below, ThermoCalc predicts that only the WN nitride phase is stable up to $\approx 600 \mathrm{~K}$. Below $600 \mathrm{~K}$ any excess $\mathrm{N}$ not bound in the WN phase degasses from the surface and above $600 \mathrm{~K}$ the nitride decomposes and all $\mathrm{N}$ degasses from the surface.

Therefore, from a thermodynamic point of view one expects that $\mathrm{N}$ accumulation in $\mathrm{W}$ due to ion implantation is low: At low temperatures, due to the low diffusion, $\mathrm{N}$ accumulation is limited to the implantation zone which saturates at a given concentration ( $50 \%$ for the WN phase). At higher temperatures diffusion is high but the nitride phases are unstable and out-diffusion of nitrogen limits the accumulated amount.

\section{Experiment}

\subsection{Sample preparation}

The samples used in these experiments were thin W (nominally $500 \mathrm{~nm}$ ) layers on 10x12 mm² Si substrates with a nominally $100 \mathrm{~nm}$ thick $\mathrm{Cu}$ interlayer to compensate stresses between the Si substrates and the W layer. These multilayered samples were produced by de magnetron deposition using Ar as process gas. Prior to deposition the Si substrates were etched with Ar for 2 minutes (25 sccm Ar, $100 \mathrm{~W} \mathrm{RF}, 4.1 \times 10^{-3} \mathrm{mbar}$ ) to clean the surface. Then the $\mathrm{Cu}$ layer was deposited during 8.5 minutes $(25 \mathrm{sccm}$ Ar, $\left.100 \mathrm{~W} \mathrm{RF}, 4.1 \times 10^{-3} \mathrm{mbar}\right)$. Finally the $\mathrm{W}$ layer was deposited during 64 minutes (25 sccm Ar, $100 \mathrm{~W}$ DC, $4.1 \times 10^{-3}$ mbar). To assure a homogenous layer thickness across the sample the substrates were rotated during the deposition process. A subsequent Rutherford Backscattering (RBS) analysis of the samples prepared in that manner showed that the layers were homogenous within the accuracy (see below in section 3.3) of the RBS measurement. 


\subsection{Nitrogen plasma exposure}

The $\mathrm{N}$ accumulation as function of energy and fluence and the sputtering of $\mathrm{W}$ by $\mathrm{N}$ was investigated in a low-pressure steady- state ECR plasma chamber "PLAQ" [8]. The ion energy was varied by applying a DC bias voltage in the range from $20 \mathrm{~V}$ to $500 \mathrm{~V}$ to the targets. The ECR plasma is ignited inside a metal cage $(150 \mathrm{~mm}$ in height, $140 \mathrm{~mm}$ in diameter). Particles can leave the cage in an axial direction through a hole in the bottom plate with a diameter of $55 \mathrm{~mm}$. A tungsten mesh prevents microwave radiation from leaving the cage which assures that the samples are not heated by microwaves and maintain a temperature of $\approx 350 \mathrm{~K}$ as measured by calibrated thermography. A diverging plasma beam impinges perpendicularly onto the substrates which are located $100 \mathrm{~mm}$ below the cage exit. To modify the energy of the incoming ions the substrate holder was biased with d.c. voltages up to $-500 \mathrm{~V}$. A metal shutter can be used to block the plasma beam hitting the substrates. Prior to the exposures the discharge was ignited with the shutter closed for $\approx 60$ minutes to minimize co-bombardment with impurity ions such as water or oxygen and to ensure reproducible conditions.

Prior to the exposures the ECR plasma has been extensively diagnosed. The ionflux and ion-energy distribution were measured with a differentially pumped retarding field analyzer (RFA). The composition of the ion flux to the sample was measured with a plasma monitor. The RFA used in the ion energy calibration measurements had the same shape as the sample holder in the later implantation experiments to assure that the plasma was identical in both cases. The whole RFA can be biased to some negative potential $U_{A c c}$ to vary the energy in the incident ions from the plasma. All voltages inside the RFA float on this external acceleration voltage $U_{A c c}$. The ions from the plasma are collected by a $(100 \mu \mathrm{m})$ pin hole in the center of the RFA. Behind the pin hole three grids are located: The first grid is biased at a constant negative voltage of $-54 \mathrm{~V}$ to repel electrons that have entered through the pin hole so that only the ion current is measured. The second and third grids were kept at the same variable potential $U_{\text {Disc }}$ which thus discriminates the ions that can pass through by their energies. Behind all three grids a small Faraday cup measures the ions that have penetrated the grid system. By taking the derivative of the Faraday cup current as function of $U_{\text {Disc }}$ the ion energy distribution is derived.

The resulting ion energy distributions for different values of $U_{A c c}$ are shown in Fig. 2. None of the energy distributions exhibit a pronounced low energy tail indicating that the sheath is collisionless. The energy of the ions is determined by the plasma potential $U_{\text {Plasma }}$ at their place of origin, plus the drop over the sheath or $U_{A c c}$ potential. In addition to the acceleration by these potential drops, the ions are also accelerated by the expanding magnetic field outside the ECR ionization zone. Most of the ions $(\approx 70 \%)$ are in the low energy peak and have the energy expected from the acceleration by the sheath or $U_{A c c}$ voltage. These low energy ions are produced close to the target by charge exchange collisions and are thus only accelerated by the sheath or $U_{A c c}$ voltage [9]. The smaller high energy peak can be attributed to ions that have not undergone any collisions and therefore carry the full energy of $U_{\text {Plasma }}+$ sheath or $U_{\text {Acc }}[9]$.

Form the known size of the sampling aperture $(100 \mu \mathrm{m})$ and the geometrical 
transmission of the grids inside the analyzer (75\%) the absolute flux density was determined from the integral over each of these peaks. This procedure yields the ion flux in units of $\left(\frac{\mathrm{ions}}{\mathrm{m}^{2} \mathrm{~s}}\right)$ for the respective bias voltage. For the given discharge pressures of $0.25,0.5$, and $1 \mathrm{~Pa}$ and a nominal microwave power of $150 \mathrm{~W}$ the mass-integrated ion flux was $3.27 \cdot 10^{19} \mathrm{~m}^{-2} \mathrm{~s}^{-1}$ for floating substrate, $3.73 \cdot 10^{19} \mathrm{~m}^{-2} \mathrm{~s}^{-1}$ for $-100 \mathrm{~V}$ bias and $4.97 \cdot 10^{19} \mathrm{~m}^{-2} \mathrm{~s}^{-1}$ for $-500 \mathrm{~V}$. The RFA measurements were repeated several times to assure that the flux measurement was reproducible.

While the RFA measurements give information about the ion energy, the ion mass remains unknown. Therefore a plasma monitor (Hiden Analytical EQP 300) was used to measure mass distribution of the ions leaving the plasma at the substrate position. Due to technical limitations the plasma monitor could not be biased to the acceleration potential $U_{A c c}$, but had to be operated at floating potential. The ions are sampled through a $250 \mu \mathrm{m}$ hole in the floating substrate holder. The floating potential of the substrate holder dummy ranged from -2.4 to $-3 \mathrm{~V}$. To maintain constant energy resolution ions are either accelerated or decelerated to a constant pass energy through the $45^{\circ}$ sector field. The voltage of the first element of the extraction lens (extraction) was set to $-5 \mathrm{~V}$ and the second element (lens1) at $-189 \mathrm{~V}$ according to the recommendations by Hamers et al. to avoid chromatic abberation [10]. The mass-dependent transmission was assumed to be constant.

For long-term stability, measurements were conducted with the Faraday detector. For pressures between 0.25 and $1 \mathrm{~Pa}$ the ion flux consisted dominantly of $\mathrm{N}_{2}^{+}$ions $(\approx 80-90 \%)$ with minor contributions of $\mathrm{N}_{3}^{+}$and $\mathrm{N}^{+}(\approx 5-10 \%$ each $)$. To assure identical conditions during the characterization and the implantation experiments the total current onto the substrate holder and the optical emission of the plasma in the visible range were monitored.

Additional implantation experiments investigating the temperature dependence of $\mathrm{N}$ accumulation in $\mathrm{W}$ were performed at the IOM Leipzig. In a plasma immersion implantation experiment solid W samples were exposed to a low-pressure, lowtemperature nitrogen plasma at a pressure of $0.6 \mathrm{~Pa}$ and an electron temperature of $1 \mathrm{eV}$. A pulsed $10 \mathrm{kV}$ bias was applied to the $\mathrm{W}$ samples with a repetition frequency between 1.2 and $4.2 \mathrm{kHz}$ and a pulse length of $15 \mu \mathrm{s}$. The samples were heated by these pulses to temperatures ranging from $600 \mathrm{~K}$ to $750 \mathrm{~K}$. The particle flux in this experiment consisted of the low energy ion flux $\left(10^{20} \mathrm{~m}^{-2} \mathrm{~s}^{-1}\right)$ from the plasma plus the high energy ion flux $\left(10^{18} \mathrm{~m}^{-2} \mathrm{~s}^{-1}\right)$ during the pulses. The low energy flux was dominated by $\mathrm{N}_{2}^{+}$ions $(\approx 90 \%), \mathrm{N}^{+}$ions were the minority species with a flux fraction of $\approx 10 \%$. In between pulses the ions from the low energy background flux were only accelerated by the sheath potential having energies $<5 \mathrm{eV} / \mathrm{N}$. Due to their higher implantation range the $\mathrm{N}$ accumulation in the surface should be governed by the ions accelerated by the $10 \mathrm{kV}$ bias. The atomic/molecular ion ratio during this $10 \mathrm{kV}$ pulse phase is identical to the original plasma composition as comparatively long pulses were employed. Therefore, for to the $\mathrm{N}_{2}^{+}$majority species, the energy was $5 \mathrm{keV} / \mathrm{N}$. 


\subsection{Ion beam analysis}

Ion beam analysis was used to determine the $\mathrm{N}$ accumulation in $\mathrm{W}$ and to measure the thickness change of the $\mathrm{W}$ layers due to sputter erosion. For the measurement of the N content nuclear reaction analysis (NRA) was applied using the ${ }^{14} \mathrm{~N}\left({ }^{3} \mathrm{He}, \mathrm{p}\right){ }^{16} \mathrm{O}$ reaction. An energy of $3.8 \mathrm{MeV}$ of the probing ${ }^{3} \mathrm{He}$ ion beam was chosen such that the cross section [11] for the nuclear reaction showed only little variation with respect to the ${ }^{3} \mathrm{He}$ energy, resulting in a constant detection efficiency over the implantation depth of the $\mathrm{N}$ ions. The protons from the nuclear reaction were counted in a detector with a solid angle of $29.95 \mathrm{sr}$. The ${ }^{14} \mathrm{~N}\left({ }^{3} \mathrm{He}, \mathrm{p}\right){ }^{16} \mathrm{O}$ reaction produces protons of at least 7 different energies due different excitation levels of the compound core during the reaction. We used the sum of the counts in $\mathrm{p} 1+\mathrm{p} 2$ peaks. They cannot be separated in the spectrum and are the strongest peaks from this reaction. They are also well separated from the peaks from other nuclear reactions in the spectrum. To convert the proton counts to a $\mathrm{N}$ areal density, a thin ZrN layer on Si was used as a calibration target. For the calibration the $\mathrm{N}$ content in the thin ZrN layer was first measured using RBS for which the elastic scattering cross sections can be calculated. Then an NRA spectrum of the ZrN layer was measured using $3.8 \mathrm{MeV}^{3} \mathrm{He}$ as described above and from the comparison of the proton counts with the N content measured by RBS a calibration factor was determined. The ZrN layer was thin enough such that the energy loss in the layer did not result in a significant variation of the nuclear reaction cross section across the layer. An analysis dose of $40 \mu \mathrm{C}$ was accumulated for each spectrum to assure good counting statistics at acceptable measurement times. Still the sensitivity of NRA to detect $\mathrm{N}$ is low: 0.13 counts $/ \mu \mathrm{C}$ for $10^{15} \mathrm{~N} / \mathrm{cm}^{2}$.

To measure the $\mathrm{W}$ erosion by $\mathrm{N}$ the change in the areal density of the $\mathrm{W}$ layer was measured by RBS using a $2.3 \mathrm{MeV}{ }^{4} \mathrm{He}$ ion beam. Due to the high elastic scattering cross section of $W$, this measurement is very sensitive to changes in the $W$ areal density in the layer. Changes of the order of $10^{13} \mathrm{~W} / \mathrm{cm}^{2}$ can be detected.

\subsection{XPS measurements}

To investigate the formation of nitride phases during implantation XPS measurements on N-implanted $\mathrm{W}$ samples were performed. The measurements were conducted in a PHI ESCA 5600 XPS system using monochromated Al K $\alpha$ radiation ( $h \nu=1486.6 \mathrm{eV}$ ). The hemispherical analyzer was operated in FAT mode with pass energies of $2.95 \mathrm{eV}$ for high-resolution spectra and $93.9 \mathrm{eV}$ for survey scans. The binding energy (BE) axis is referenced to the $\mathrm{Au} 4 f_{7 / 2}$ peak at $84.0 \mathrm{eV}$ and the spectra are measured at $45^{\circ}$ exit angle. Further experimental details can be found elsewhere [12]. Since XPS is very surface sensitive the $\mathrm{N}$ implantation is performed in situ to avoid contamination of the surface with adsorbates during transport through air. In the XPS system the N implantation was performed using a ion gun (Specs IQE 12/38) with $1 \mathrm{kV}$ acceleration voltage yielding a total ion current of $\approx 0.5 \mu \mathrm{A}$. The ion beam is scanned over an area of $\approx 2 \mathrm{x} 2 \mathrm{~mm}^{2}$ to achieve a homogeneous implantation profile. Since the ions are not mass separated, the exact ion energy and fluence are unknown, because the flux fractions of the individual molecular ions from the source are unknown. For the nitrogen fluence 
calculations we therefore assume a pure $\mathrm{N}_{2}^{+}$beam. No impurity ions are emitted from the source. This was verified by the post N-implantation XPS spectra which only showed $\mathrm{N}$ and $\mathrm{W}$ lines and no other impurities, in particular no oxygen contamination.

The literature on XPS peak shifts due to $\mathrm{W}$ nitride formation and $\mathrm{N}_{2}$ adsorption on tungsten is fairly limited. Molecular nitrogen adsorbs on W surfaces only below $100 \mathrm{~K}$. Adsorbed $\mathrm{N}_{2}$ leads to $\mathrm{N} 1 s$ peaks around $400.0 \mathrm{eV}$, with an additional broad shake-up peak approximately $5.5 \mathrm{eV}$ at higher Binding energies (BEs) $[13,14,15]$. The N $1 s$ peak for tungsten nitrides is shifted to lower BEs. Values reported in the literature vary from $397.0 \mathrm{eV}[16], 397.3 \mathrm{eV}[17,18], 397.5 \mathrm{eV}$ [19], to $397.7 \mathrm{eV}$ [20]. For the $\mathrm{W}$ 4f peak more data is available in the literature. The nitrides generally exhibit slightly higher binding energies than the metallic $\mathrm{W} 4 f_{7 / 2}$ peak at $31.4 \mathrm{eV}$. Nevertheless, also here some scatter in the available data is noticed. Absolute BE values for the $\mathrm{W} 4 f_{7 / 2}$ peak are given as $32.3 \mathrm{eV}$ [21], 31.17 and $31.42 \mathrm{eV}$ for different nitride stoichiometries [18], 32.1 eV [22]. Values as high as 32.8 and $33.2 \mathrm{eV}$ [16], $32.9 \mathrm{eV}$ [17], and $33.0 \mathrm{eV}$ [23] are also given in literature. A relative shift of $+0.3 \mathrm{eV}$ is reported in [24] for nitrogen ions implanted into polycrystalline tungsten.

Both the $\mathrm{W} 4 f$ and the $\mathrm{N} 1 s$ peaks were measured during the implantation steps of nitrogen ions as A function of the ion fluence. The implantations were performed at 300, 600 and $800 \mathrm{~K}$ substrate temperature. Prior to the XPS measurements, the samples were allowed to cool down to $300 \mathrm{~K}$. To confirm that no modifications in the nitrogen inventory occurred during the cooling, survey spectra were measured both at elevated temperatures directly after implantation and after cooling to $300 \mathrm{~K}$, both for several implantation steps at $600 \mathrm{~K}$ and $800 \mathrm{~K}$. Quantitative analysis of the nitrogen contents measured at high temperature and at $300 \mathrm{~K}$ showed only deviations within the statistical error. Therefore, no quantitative changes occurred between implantation at elevated temperatures and the XPS measurements at $300 \mathrm{~K}$.

\section{Modeling}

The accumulation of $\mathrm{N}$ in $\mathrm{W}$ and the sputter yield of $\mathrm{W}$ due to $\mathrm{N}$ bombardment were modeled using the Monte Carlo code TRIDYN [25]. TRIDYN simulates the interaction of fast particles with amorphous solids by approximating the three dimensional trajectories of the projectiles and recoils as a succession of binary collisions. It can be operated in a static mode in which the target is not modified during the simulated bombardment and in a dynamic mode where the target is modified in composition and thickness due to sputter erosion of the target surface and deposition of the incident species. The most sensitive parameter for sputter calculations using TRIDYN or similar programs is the surface binding energy. While the surface binding energy (SBE) for pure target surfaces is usually taken as the heat of sublimation, it is essentially a fit parameter for mixed surfaces. In the calculations shown here the surface binding energy of $\mathrm{N}$ and $\mathrm{W}$ was kept constant at the value for pure $\mathrm{W}$ of $8.68 \mathrm{eV}$. 


\section{Results and discussion}

The accumulation of $\mathrm{N}$ in $\mathrm{W}$ as function of energy and fluence was investigated after the implantations performed in the PLAQ low-temperature ECR plasma. In Fig. 3 the accumulated $\mathrm{N}$ amount as function of the $\mathrm{N}$ ion fluence, determined from NRA is shown for three different target bias voltages: 20,50 and $100 \mathrm{~V}$. Assuming that the N plasma consisted mainly of $\mathrm{N}_{2}^{+}$ions these correspond to 10,25 and $50 \mathrm{eV} / \mathrm{N}$. The retained amount of $\mathrm{N}$ in the $\mathrm{W}$ surface quickly saturates with fluence. This fast saturation is in line with the predictions derived from the available thermodynamic data (see section 2): Due to the low diffusion no appreciable transfer of $\mathrm{N}$ into the bulk is expected, therefore only the implantation range is saturated with a maximum $\mathrm{N}$ concentration determined by one of the nitride phases (max. $50 \%$ for the WN phase). Also shown in Fig. 3 are three TRIDYN simulations of the accumulation of $\mathrm{N}$ in $\mathrm{W}$ at low temperatures. To model the accumulation TRIDYN was used in dynamic mode: The modification of the $\mathrm{W}$ target surface composition due to $\mathrm{W}$ erosion and $\mathrm{N}$ implantation was taken into account. As explained in section 4 it is reasonable to assume that all $\mathrm{N}$ not bound in a nitride phase sublimes from the surface resulting in a maximum $\mathrm{N}$ surface concentration of $50 \%$. Therefore the maximum concentration of $\mathrm{N}$ was limited to $50 \%$ in the dynamic TRIDYN calculations. In Fig. 3 both the experimental and the TRIDYN calculations show the same quick saturation of the surface $\mathrm{N}$ amount. Also the levels predicted by TRIDYN are rather similar to those found in the experiment. Assuming that all $\mathrm{N}$ is bound in a WN phase with $50 \% \mathrm{~N}$, the thickness $\Delta x$ of this WN layer can be calculated from the maximum $\mathrm{N}$ areal density of $\delta_{\text {Max }} \approx 8 \cdot 10^{19} \mathrm{~m}^{-2}$ found for $100 \mathrm{~V}$ bias using the density of pure WN: $\rho \approx 9 \cdot 10^{28} \mathrm{~m}^{-3}[26]$.

$$
\Delta x=\frac{0.5 \rho}{\delta_{\text {Max }}} \approx 1.8 \cdot 10^{-9} \mathrm{~m}
$$

This value for $\Delta x$ is comparable to the implantation range of the $\mathrm{N}$ for $100 \mathrm{~V}$ bias. This confirms our assumption that the nitride is only formed within the implantation range and that diffusion does not occur at near room temperature implantations.

The temperature dependence of the $\mathrm{N}$ accumulation in $\mathrm{W}$ was investigated in the plasma immersion implantation experiments at IOM Leipzig. The W samples were heated in the temperature range from 600 to $750 \mathrm{~K}$. The samples were implanted dominantly with $\mathrm{N}_{2}^{+}$ions accelerated by $10 \mathrm{kV}$ pulses at similar fluences of several $10^{22} \mathrm{~m}^{-2} \mathrm{~s}^{-1}$. The resulting $\mathrm{N}$ accumulation as function of temperature in these samples as measured by NRA is shown in Fig. 4. The numbers below the data points indicate the fluence of the $\mathrm{N}_{2}^{+}$ions accelerated by the $10 \mathrm{kV}$ pulses. The amount of accumulated $\mathrm{N}$ in these samples is a factor $\approx 10$ larger than in the PLAQ despite the lower implantation fluence. The reason for this is probably the higher implantation range (by a factor of $\approx 15$ ) of the $\mathrm{N}_{2}^{+}$ions accelerated by the $10 \mathrm{kV}$ pulses which can therefore saturate a larger depth range in the $\mathrm{W}$ surface. For temperatures above $700 \mathrm{~K}$ the accumulated amount of $\mathrm{N}$ decreases despite the slightly higher implantation fluence for the high temperature points. Again this observation is line with the prediction based on the available thermodynamic data in section 2: Above $600 \mathrm{~K}$ the nitrides become instable and $\mathrm{N}$ is lost from the $\mathrm{W}$ surface by sublimation. 
To investigate the physical sputtering of $\mathrm{W}$ by $\mathrm{N}, \mathrm{W} / \mathrm{Cu} / \mathrm{Si}$-substrate samples were bombarded by a $\mathrm{N}$ plasma in the PLAQ setup with a DC sample bias varying from $50 \mathrm{~V}$ to $500 \mathrm{~V}$, corresponding to $25 \mathrm{eV} / \mathrm{N}$ to $250 \mathrm{eV} / \mathrm{N}$ for a predominantly $\mathrm{N}_{2}^{+}$ion producing plasma. The samples were bombarded with a fluence $\Gamma_{\text {Imp }} \approx 10^{23} \mathrm{~m}^{-2}$ which according to Fig. 3 results in a $\mathrm{N}$ saturated implantation zone. Thereby $\Gamma_{\text {Imp }}$ is the actual $\mathrm{N}$ fluence $\mathrm{m}^{-2}$ which is calculated from the ion fluence based on the molecular ion flux fractions determined during the plasma monitor measurements. The sputter yields were then determined by dividing the change in the W areal density $\Delta \delta_{W}$ in the W layer (as determined by RBS) by $\Gamma_{\text {Imp }}$.

The so-determined sputter yield is compared to calculated sputter yields of W by $\mathrm{N}$ ions and to experimental data for sputtering of $\mathrm{W}$ by $\mathrm{Ne}$ in Fig. 5. To model the sputtering of $\mathrm{W}$ by $\mathrm{N}$, TRIDYN was both used in static and dynamic mode. In all TRIDYN calculations the particle spectrum of the impacting $\mathrm{N}$ ions was taken from plasma monitor measurements: $5 \% \mathrm{~N}^{3+}, 85 \% \mathrm{~N}^{2+}$ and $10 \% \mathrm{~N}^{+}$. In the static calculation the sputter yield $Y_{\text {Static }}$ of $\mathrm{W}$ by $\mathrm{N}$ for a constant pure $\mathrm{W}$ surface was calculated not taking into account the accumulation of $\mathrm{N}$. The resulting $Y_{\text {Static }}$ is shown in Fig. 5 as connected open circles. $Y_{\text {Static }}$ is much higher than the experimentally observed yield, shown as connected solid star symbols in Fig. 5. The connected open triangle symbols in Fig. 5 show the result of dynamic TRIDYN calculation which allowed the accumulation of up to $50 \%$ of $\mathrm{N}$ in the $\mathrm{W}$ surface. Due to the resulting lower $\mathrm{W}$ surface concentration the calculated partial $\mathrm{W}$ sputter yield $Y_{D y n}$ is now lower than $Y_{\text {Static }}$ and quite close to the experimental data particularly for the higher energies.

The sputtering of $\mathrm{W}$ by $\mathrm{N}$ ions extracted from the low- temperature plasma can be understood by taking into account the different molecular ion species from the plasma and the effect of $\mathrm{N}$ accumulation in the surface. The good agreement between the experimental data and the dynamic TRIDYN simulation results also indicates that to choose the SBE of pure $\mathrm{W}$ for the SBE of $\mathrm{W}$ and $\mathrm{N}$ in a mixed surface, as explained in section 4, was a valid assumption. It further indicates that there is no enhanced erosion of $\mathrm{W}$ from a $\mathrm{N}$ and $\mathrm{W}$ mixed surface. The contrary is the case: $\mathrm{W}$ erosion is reduced by the accumulation of $\mathrm{N}$ in surface. This apparent reduction in $\mathrm{W}$ erosion could be a reason for the improved plasma performance found in ASDEX Upgrade for discharges with $\mathrm{N}$ puffing. Other puffing species like $\mathrm{Ar}$, Ne or even $\mathrm{H}$ can only reach surface concentrations in the \% level, which does not reduce the $\mathrm{W}$ sputter yield. However, $\mathrm{N}$ can reach levels of up to $50 \%$, thus reducing the apparent erosion of $\mathrm{W}$. Therefore, in contrast to $\mathrm{N}$ the other noble gas puffing species enhance the sputter erosion of $\mathrm{W}$ due their higher mass compared to a pure $\mathrm{H}$ plasma. This is also apparent from a comparison of $\mathrm{Ne}$ and $\mathrm{N}$ sputtering data in Fig. 5: The Ne sputtering yield is much higher despite the small mass difference between Ne and N. Also the experimental Ne sputter yield closely matches the static TRIDYN calculation for a pure W surface which again shows that Ne accumulation in the W surface is so low that it does not affect the partial sputter yield of $\mathrm{W}$. Thus $\mathrm{N}$ as a seeding species is the better choice over $\mathrm{Ne}$ or even Ar.

From the XPS measurements after $\mathrm{N}_{2}^{+}$implantation into tungsten at 300, 600, and $800 \mathrm{~K}$, clearly the formation of tungsten nitride is visible. In both binding energy 
regions $\mathrm{W} 4 f$ and $\mathrm{N} 1 s$, the peaks are located at positions which correspond to the available literature data for tungsten nitrides (references see section 3.4). Fig. 6 and Fig. 7 show the respective XPS spectra for $\mathrm{N}$ fluences at which the W/N XPS signal ratio reached equilibrium. The respective $\mathrm{N}$ fluences for the spectra at 300,600 , and $800 \mathrm{~K}$ are: $4.2,5.4$, and $4.6 \times 10^{18} \mathrm{~m}^{-2}$. The spectra in Fig. 6 are fitted with asymmetric Gauss-Lorentz functions and a linear background is applied. The shape parameters (tail scale and length, Gauss-Lorentz ratio, full-width-at-half-maximum) of the $\mathrm{W} 4 f$ peaks determined at the clean $\mathrm{W}$ substrate are fixed as starting parameters for the fits performed after ion implantation with an additional doublet at higher binding energies. For this doublet, the area ratio between the $7 / 2$ and $5 / 2$ peaks and their separation are taken from the doublet for metallic $\mathrm{W}$ and kept fixed during the fit procedure. All other parameters, in particular the peak position and the height, are fitted. The results of the fitting procedure are shown in Fig. 6. The experimental data points are plotted as open circles. The doublet assigned to metallic $\mathrm{W}$ is drawn in broken lines, the doublet assigned to the nitride and the sum of all peaks (and the linear background) are plotted as solid lines. The fine dashed line indicates the deviation of the sum of all fit functions to the data points. As can be seen, the fit is quite satisfying. The position of the metallic $\mathrm{W} 4 f_{7 / 2}$ peak is at $31.40_{-0.04}^{+0.02} \mathrm{eV}$ and can be accepted as constant within the experimental accuracy (energy step width in the spectra: $0.025 \mathrm{eV}$ ). The $\mathrm{W} 4 f$ peaks are well reproduced by adding a second doublet which is assigned to tungsten nitride. For the implantation after 300 and $600 \mathrm{~K}$, the separation between the two $\mathrm{W} 4 f$ peaks is $0.45 \pm 0.03 \mathrm{eV}$. With a binding energy of $31.85 \pm 0.03 \mathrm{eV}$ this binding energy corresponds to the range of binding energies in the literature (see section 3.4), however, it is at the lower end of the range. After implantation at $800 \mathrm{~K}$, the intensity assigned to nitride at higher binding energies is shifted by $0.90 \mathrm{eV}$ with respect to the metallic peak. Since this shift is significantly larger than after 300 and $600 \mathrm{~K}$ implantation, it can be speculated whether the nitride has a different local atomic arrangement or whether the nitride phase has a different stoichiometry. This question, however, has to be addressed in future experiments.

The formation of the tungsten nitride during $\mathrm{N}_{2}^{+}$implantation is directly visible also in the $\mathrm{N} 1 s$ spectra, shown in Fig.7. Due to the low photoemission cross section, the $\mathrm{N} 1 s$ spectra exhibit a small signal-to-noise ratio and a detailed analysis by peak fitting is not justified. However, all signals measured after $\mathrm{N}_{2}^{+}$implantation show a peak maximum well below $400 \mathrm{eV}$ and can therefore be attributed to nitrogen bound in a tungsten nitride. The peak shapes also clearly indicate that the $\mathrm{N} 1 s$ signals consist of more than one peak. This was not described up to now in the references listed in section 3.4. These two peaks can either be the consequence of different tungsten nitride stoichiometries, or can be caused by nitrogen in different local atomic arrangements within the tungsten lattice. Since the tungsten nitride is formed by ion implantation in our experiments, local disorder due to the collision cascades is a reasonable assumption.

The quantitative analysis of the metallic and nitride peaks in the $\mathrm{W} 4 f$ signal, plotted in Fig. 8, shows that at $800 \mathrm{~K}$ only a small fraction of the tungsten within the XPS analysis region is in the nitride state. For the $300 \mathrm{~K}$ implantation, approximately $57 \%$ of the tungsten in the surface layer accessible by XPS is nitride. Without a chemical 
depth profile analysis, however, no stoichiometry can be assigned to the tungsten nitride from the XPS measurements. From Fig. 8 it can also be concluded that the amount of tungsten in the nitride state strongly decreases with the implantation temperature, reflecting the metastable nature of tungsten nitrides.

\section{Conclusions}

The interaction of $N_{2}$ plasmas and $\mathrm{N}$ ion beams with $\mathrm{W}$ surfaces was investigated. In particular, the accumulation of $\mathrm{N}$ in the $\mathrm{W}$ surface, the sputter erosion of $\mathrm{W}$ and the formation of nitride phases have been measured: It was found that the $\mathrm{N}$ content in the W surface quickly saturates once the $\mathrm{N}$ concentration within the implantation range has reached the stoichiometry of $\mathrm{W}$-nitride $(50 \% \mathrm{~N})$. Therefore to total amount of $\mathrm{N}$ in the surface depends on the $\mathrm{N}$ energy since the implantation range increases with increasing $\mathrm{N}$ energy. Due to accumulation of $\mathrm{N}$ in the $\mathrm{W}$ surface the observed partial sputter yield of $\mathrm{W}$ was significantly reduced compared to the yields expected for a pure W surface. Therefore with respect to $\mathrm{W}$ sputtering $\mathrm{N}$ is a better seeding species compared to noble gas elements which only accumulate in the surface at sub $\%$ levels which results in observed partial sputter yields equal to those for pure $\mathrm{W}$ surfaces. Implantations performed at higher temperatures indicate that WN becomes unstable above $\approx 600 \mathrm{~K}$ which is in very good agreement with thermodynamic calculations. XPS measurements after ion beam implantations showed the formation of W-nitrdes with $\mathrm{W} 4 f$ peak shifts similar to those found in literature. Also these XPS measurements show that the Wnitrides become unstable at elevated temperatures and decompose by degassing the N. In conclusion the use of $\mathrm{N}$ as a seeding species together with a $\mathrm{W}$ wall poses no problems with respect to the interaction with the first wall moreover the use of $\mathrm{N}$ leads to lower W erosion compared to noble gas seeding with Ne or Ar. 
[1] A. Kallenbach, R. Dux, M. Mayer, R. Neu, T. Pütterich, V. Bobkov, J.C. Fuchs, T. Eich, L. Giannone, O. Gruber, A. Herrmann, L.D. Horton, C.F. Maggi, H. Meister, H.W. Müller, V. Rohde, A. Sips, A. Stäbler, J. Stober, and ASDEX Upgrade Team, Nuclear Fusion 49 (2009) 045007.

[2] J. Keinonen, J. Räisänen, and A. Anttila, Applied Physics A 35 (1984) 227.

[3] E. Fromm and H. Jehn, Zeitschrift für Metallkunde 62 (1971) 378.

[4] H. Jehn and P. Ettmayer, Monatsheft Chemie 111 (1980) 1437.

[5] R. Frauenfelder, Journal of Chemical Physics 48(9) (1968) 3966.

[6] F.J. Norton and A.L. Marshall, Trans. AIME 156 (1944) 351.

[7] J.O. Andersson, T. Helander, L. Höglund, P.F. Shi, and B. Sundman, Calphad, 26 (2002) 273.

[8] B. Landkammer, A. von Keudell, and W. Jacob, J. Nucl. Mater. 264 (1999) 48.

[9] P. Reinke, W. Jacob, and W. Möller, Diamond and diamond-like films and coatings (Plenum Press, New York, 1991).

[10] E.A.G. Hamers et al., Int. J. Mass Spectrometry and Ion Processes 173 (1998) 91.

[11] L.C. McIntyre, Jr. andJ.A. Leavitt, M.D. Ashbaugh, J. Borgardt, E. Andrade, J. Rickards, and A. Oliver, Nucl. Instr. and Meth. B 118 (1996) 219.

[12] A. Wiltner, Ch. Linsmeier, and T. Jacob, J. Chem. Phys. 129 (2008) 084704.

[13] T.E. Madey, J.T. Yates Jr., and N.E. Erickson, Surf. Sci. 43 (1974) 526.

[14] J.C. Fuggle and D. Menzel, Surf. Sci. 79 (1979) 1.

[15] Q.-J. Zhang, J.C. Lin, N. Shamir, and R. Gomer, Surf. Sci. 231 (1990) 344.

[16] Y. G. Shen, Y. W. Mai, D. R. McKenzie, Q. C. Zhang, W. D. McFall, and W. E. McBride, J. Appl. Phys. 88 (2000) 1380.

[17] H.-T. Chiu and S.-H. Chuang, J. Mater. Res. 8 (1993) 1353.

[18] Ch.-W. Lee, Y.T. Kim, and S.-K. Min, Appl. Phys. Lett. 62 (1993) 3312.

[19] C. Egawa, S. Naito, and K. Tamaru, Surf. Sci. 131 (1983) 49.

[20] A. Kakanakova-Georgieva, L. Kassamakova, Ts. Marinova, R. Kakanakov, O. Noblanc, C. Arnodo, S. Cassette, and C. Brylinski, Appl. Surf. Sci. 151 (1999) 225.

[21] D.Z. Wang, J.X. Chen, H.L. Zhang, and N.K. Huang, Nucl. Instr. Meth. Phys. Res. B 171 (2000) 465.

[22] C.C. Baker and S.I. Shah, J. Vac. Sci. Technol. A 20 (2002) 1699.

[23] T. Nakajima, , K. Watanabe, and N. Watanabe, J. Electrochem. Soc. 134 (1987) 3175.

[24] I. Takano, S. Isobe, T.A. Sasaki, and Y. Baba, Appl. Surf. Sci 37 (1989) 25.

[25] W. Möller, W. Eckstein, and J. P. Biersack, Computer Physics Communications 51 No. 8 (1988) 355.

[26] Kim Soo-Hyun, Kim Jun-Ki, Kwak Nohjung, Sohn Hyunchul, Kim Jinwoong, Jung Sung-Hoon, and Hoong Mi-Ran et. al., Electrochemical and Solid-State Letters 9 (2006) C54. 


\section{Figure captions}

Fig. 1

Phase diagram of $\mathrm{N}$ and $\mathrm{W}$ calculated using the ThermoCalc ${ }^{\mathrm{TM}}$ modeling package [7]

Fig. 2

Ion energy distributions for different target bias voltages as measured by the RFA.

Fig. 3

Accumulation of $\mathrm{N}$ in $\mathrm{W}$ for low implantation temperatures as function of fluence for three different $\mathrm{N}$ ion energies.

Fig. 4

Accumulation of $\mathrm{N}$ in $\mathrm{W}$ as function of target temperature.

Fig. 5

Comparison of experimental and calculated sputter yields $\mathrm{W}$ by $\mathrm{N}$.

Fig. 6 XPS spectra of clean and $\mathrm{N}_{2}^{+}$implanted tungsten samples. The spectra are measured after fluences where the $\mathrm{W} / \mathrm{N}$ ratio is in equilibrium. Experimental data points are open circles, the fit functions for metallic $\mathrm{W}$ are plotted as broken lines. Solid lines represent the nitride peaks, the linear background and the sum of all fit functions. The deviation of the fits from the data points is shown in fine broken lines.

Fig. 7 XPS spectra in the $\mathrm{N} 1 s$ binding energy region for clean $\mathrm{W}$ and after implantation of $\mathrm{N}_{2}^{+}$at different temperatures. The raw spectra are shown without any background subtraction.

Fig. 8 Nitride peak fraction in the W $4 f$ peaks, determined by peak fitting for different implantation temperatures. 


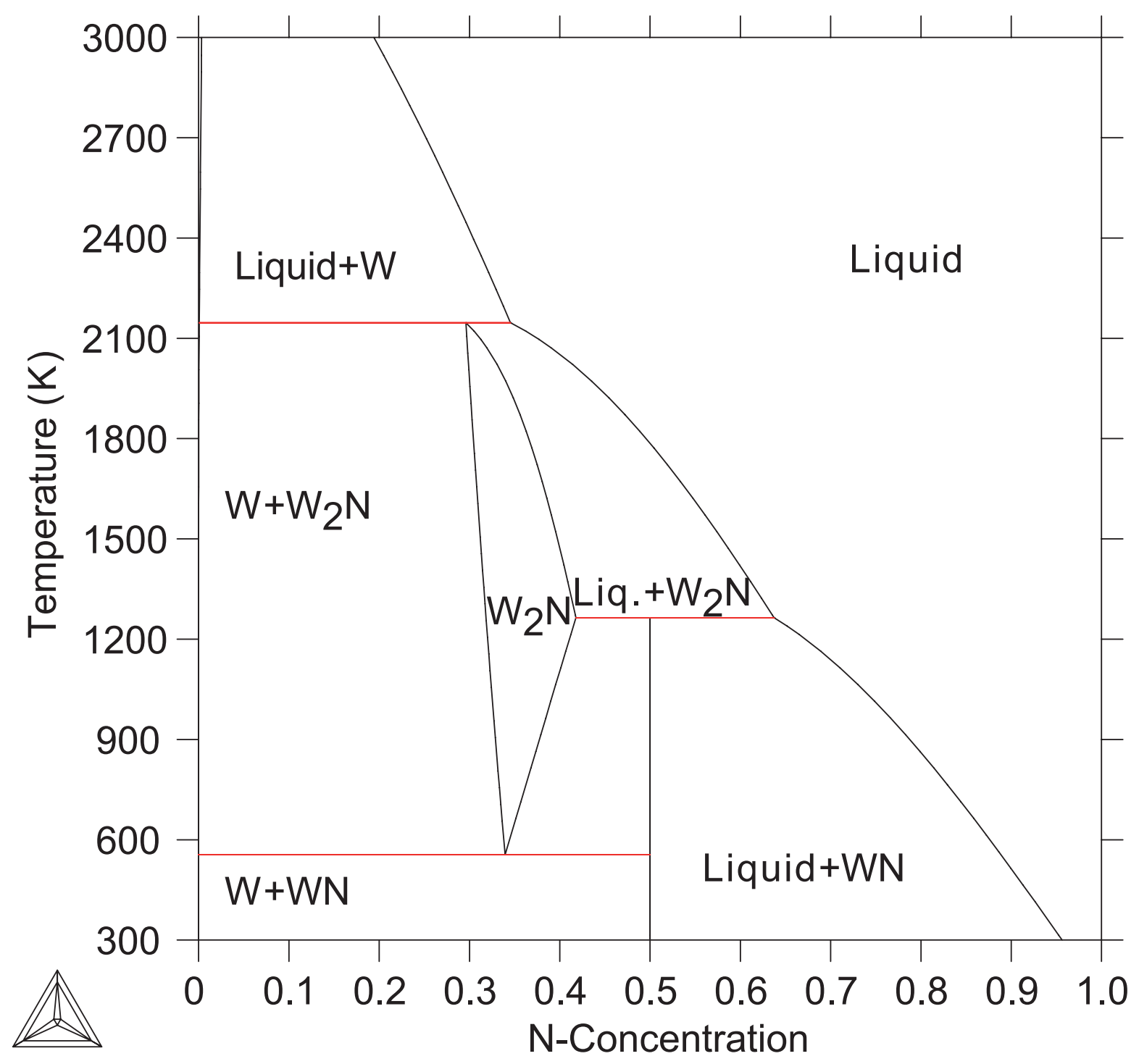

Figure 1. 


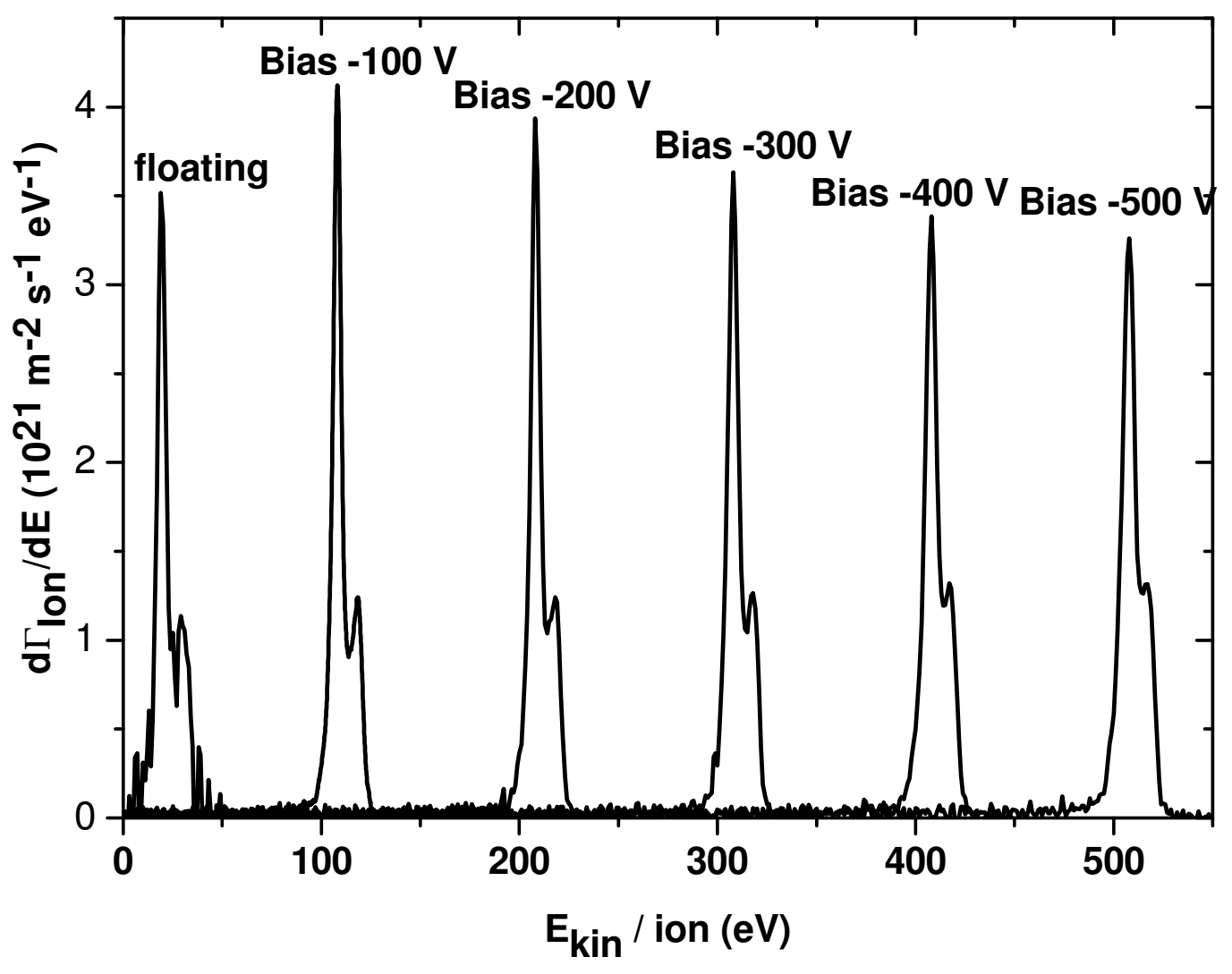

Figure 2. 


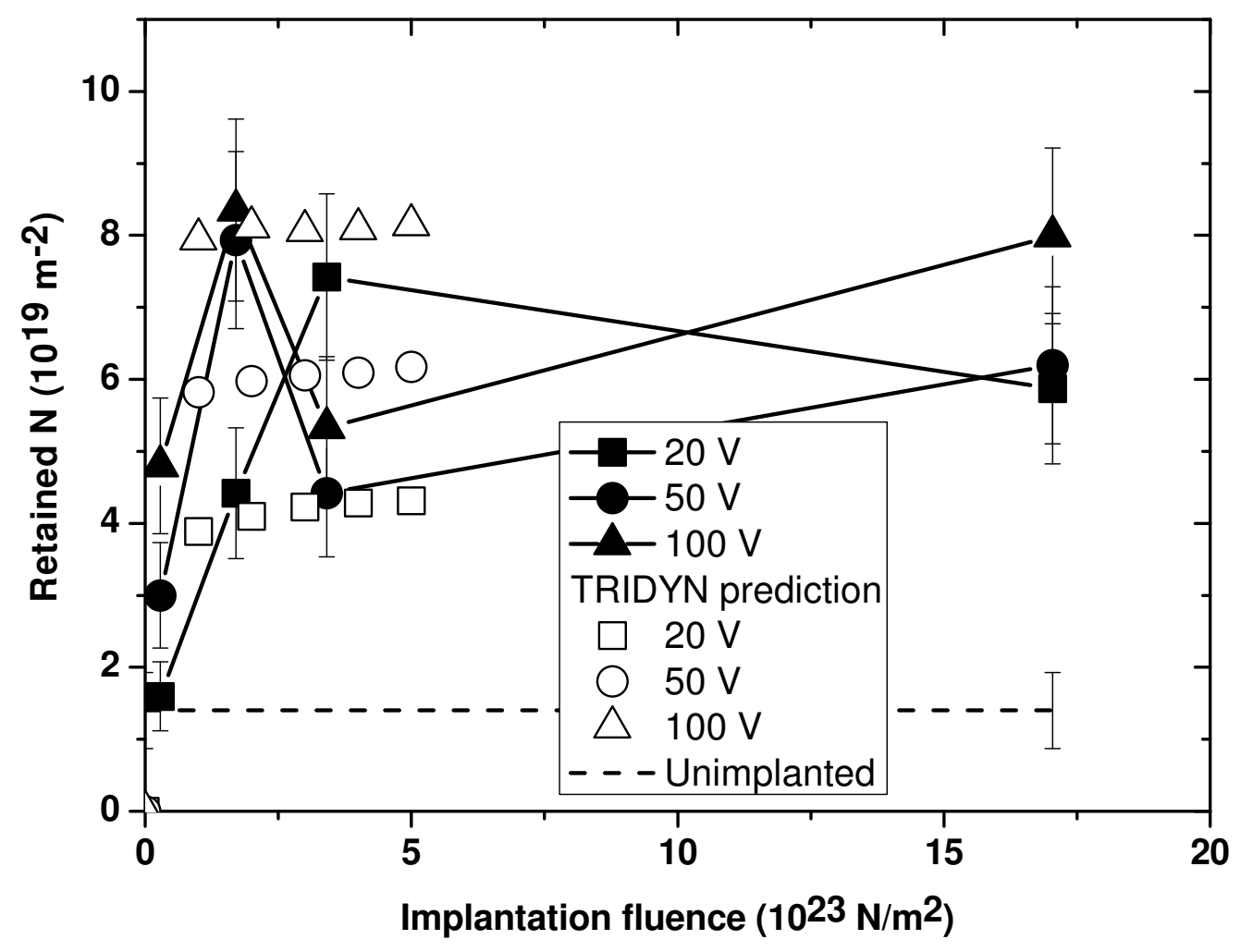

Figure 3. 


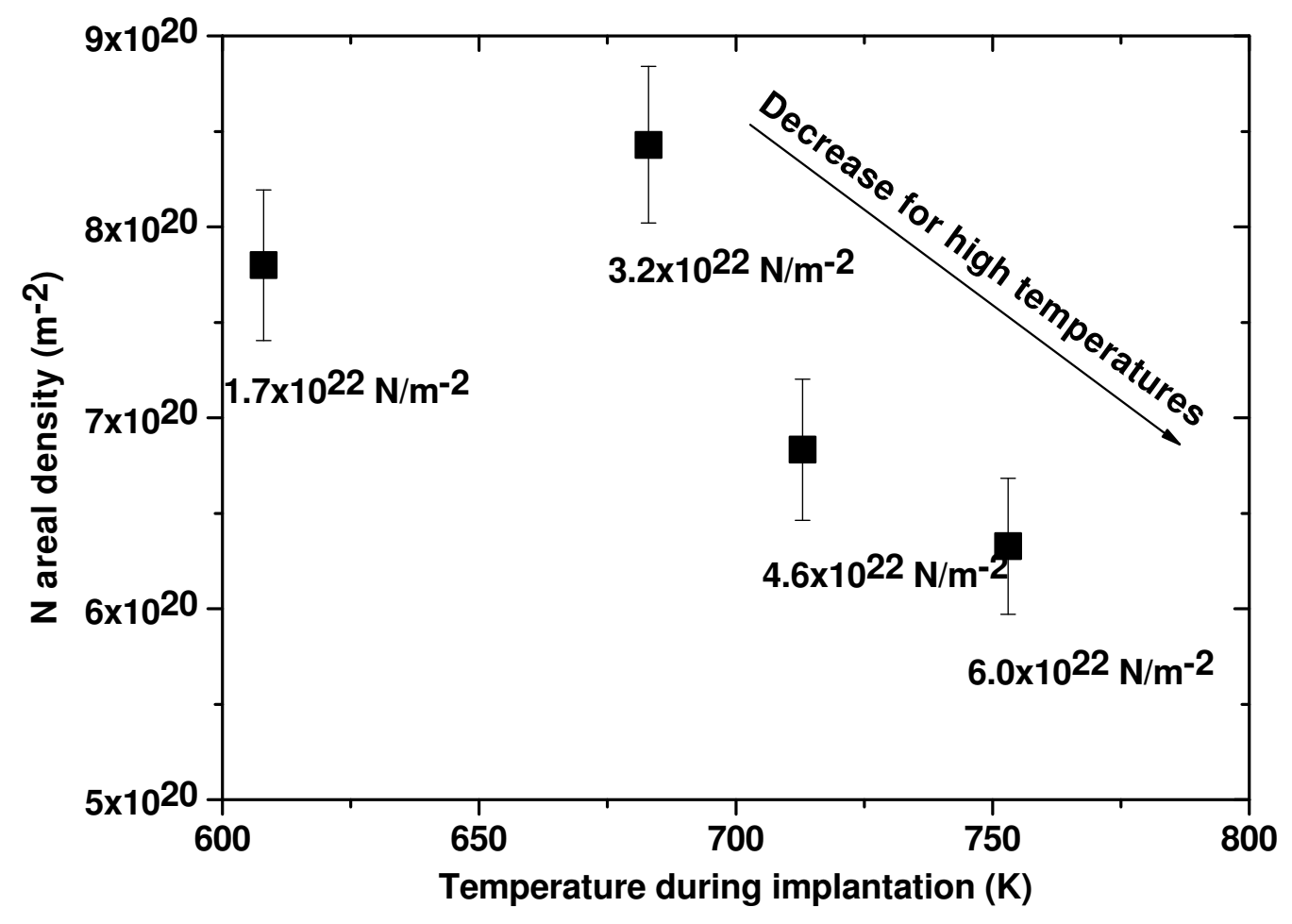

Figure 4. 


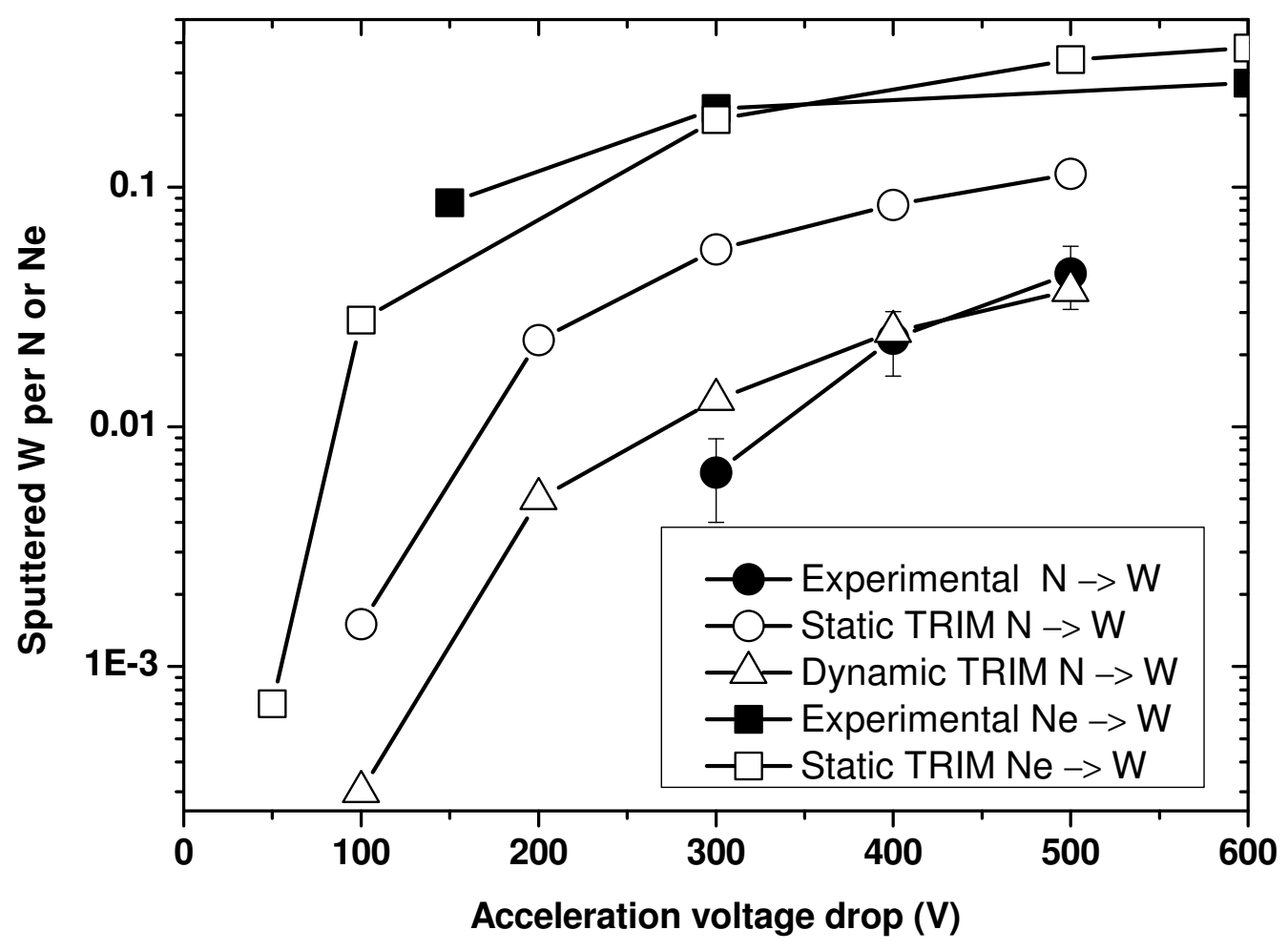

Figure 5. 


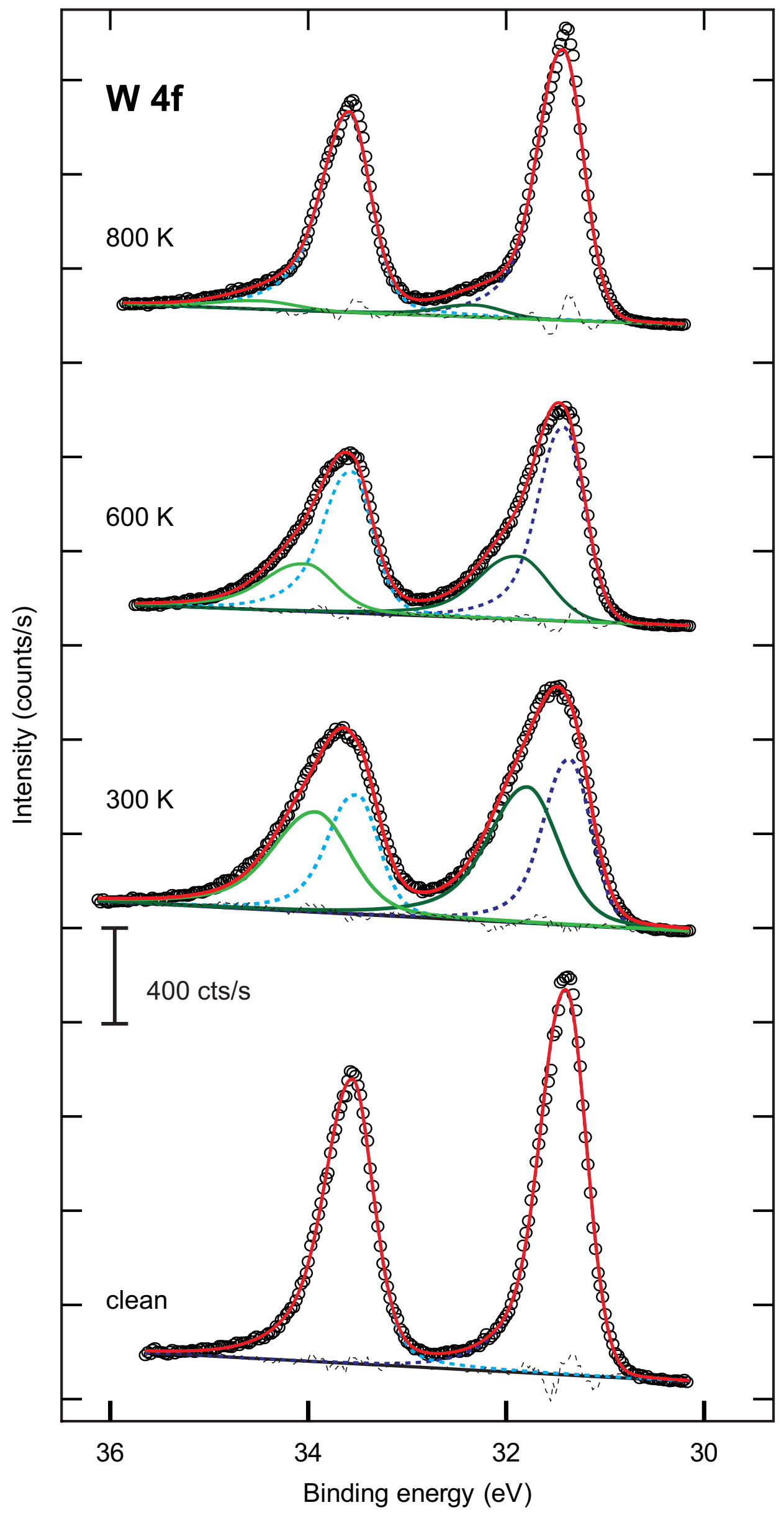

Figure 6. 


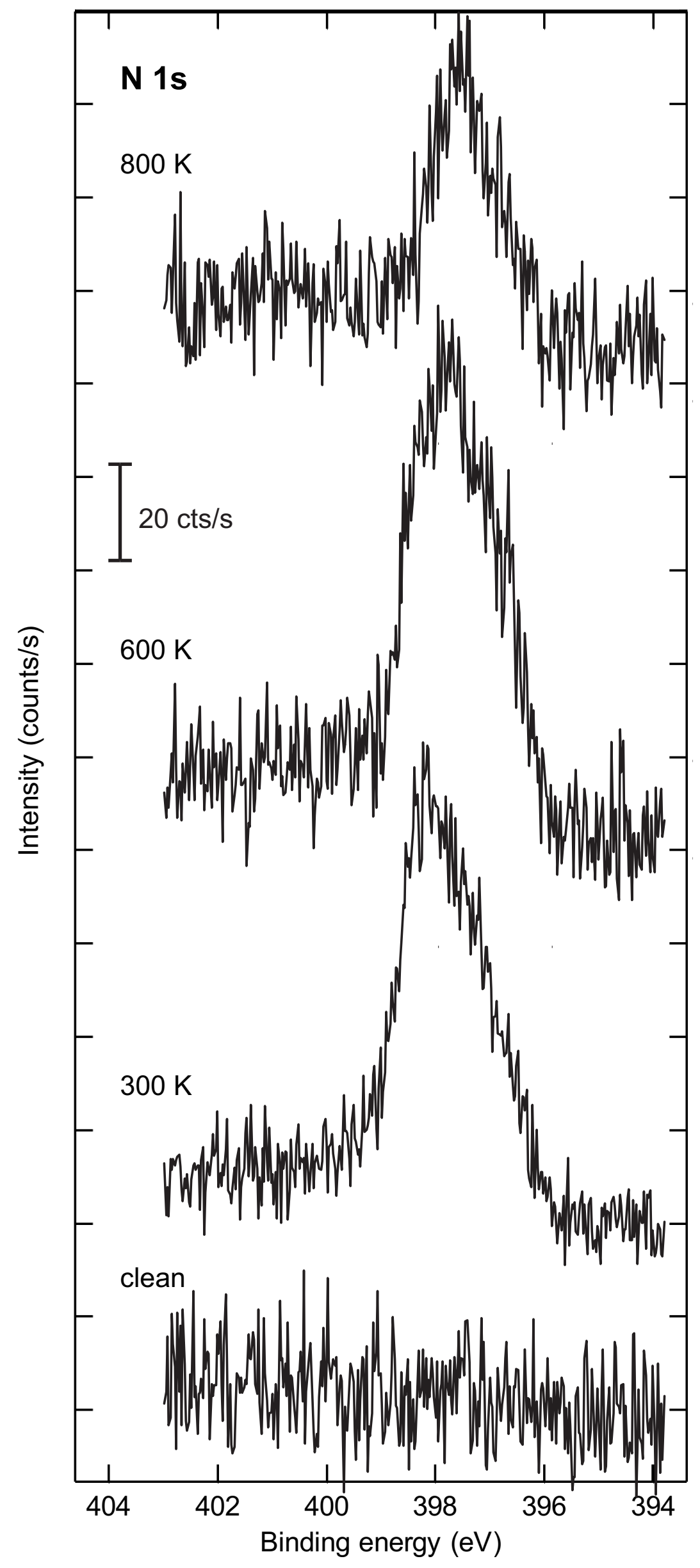

Figure 7. 


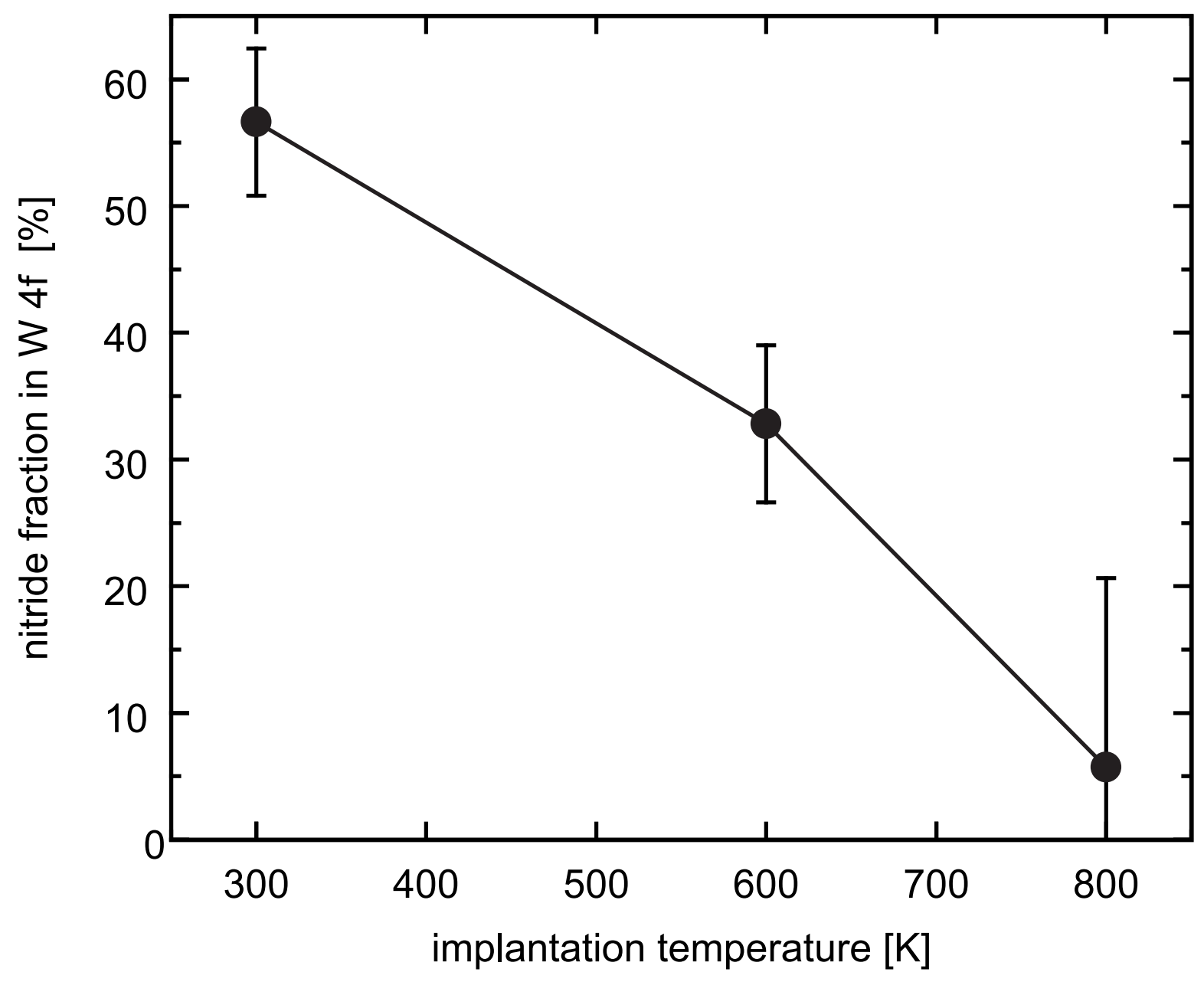

Figure 8. 